\title{
Perioperative outcomes in minimally invasive direct coronary artery bypass versus off-pump coronary artery bypass with sternotomy
}

\author{
Ali İhsan Tekin ${ }^{1}$, Ümit Arslan² \\ ${ }^{1}$ Cardiovascular Surgery Department, Kayseri Education and Training Hospital, Kayseri, Turkey \\ ${ }^{2}$ Department of Cardiovascular Surgery, Erzurum Regional Training and Research Hospital, Erzurum, Turkey
}

Videosurgery Miniinv 2017; 12 (3): 285-290

DOI: https://doi.org/10.5114/wiitm.2017.67679

\begin{abstract}
Introduction: Surgical treatment of isolated left anterior descending coronary artery disease can be performed with either minimally invasive direct coronary artery bypass via a left anterior thoracotomy (MIDCAB) or off-pump coronary artery bypass via a median sternotomy (OPCAB).

Aim: To compare the perioperative outcomes of patients undergoing MIDCAB or OPCAB surgery.

Material and methods: Patients who underwent either MIDCAB or OPCAB for isolated left anterior descending (LAD) coronary artery disease between October 2013 and December 2015 were retrospectively evaluated. Operations were carried out by the same surgical team. Preoperative, intraoperative and postoperative data of the patients were recorded for analyses.

Results: Twenty-three patients (7 females, 16 males) underwent MIDCAB surgery, and 24 patients (4 female, 20 males) underwent $O P C A B$ surgery. The two groups were comparable regarding preoperative patient characteristics. Duration of mechanical ventilation $(5.1 \pm 0.7 \mathrm{~h}$ vs. $6.6 \pm 0.9 \mathrm{~h})$, intensive care unit stay $(19.4 \pm 2.5 \mathrm{~h}$ vs. 45.8 $\pm 5.4 \mathrm{~h})$ and hospital stay $(4.3 \pm 0.4$ days vs. $5.6 \pm 0.8$ days) were significantly shorter in the MIDCAB group $(p<0.01)$. Patients in the OPCAB group required significantly more blood transfusions ( $1.83 \pm 0.38$ units vs. $0.17 \pm 0.38$ units) and fresh frozen plasma use $(2.33 \pm 0.96$ units vs. $0.69 \pm 0.76$ units) $(p<0.01)$. Conversion to sternotomy was not required in the MIDCAB group. There was no mortality, conversion to cardiopulmonary bypass or serious complication in either group.

Conclusions: We believe that the MIDCAB technique is more advantageous than the OPCAB technique in the treatment of patients with a critical $L A D$ lesion.
\end{abstract}

Key words: perioperative outcome, coronary artery bypass, left anterior descending stenosis.

\section{Introduction}

Coronary artery bypass grafting is usually applied with median sternotomy and cardiopulmonary bypass. In order to avoid complications related to cardiopulmonary bypass, off-pump coronary artery bypass (OPCAB) grafting was developed to vascularize coronary arteries through a full sternotomy on a beating heart without performing a cardiopulmonary bypass [1-3]. In recent years, however, the use of minimally invasive direct coronary bypass (MIDCAB), which does not include either cardiopulmonary bypass or full sternotomy, has been initiated in selected cases to minimize the complications associated with full sternotomy, cardiopulmonary bypass, and

\section{Address for correspondence}

Ali İhsan Tekin, Cardiovascular Surgery Department, Kayseri Education and Training Hospital, Kayseri, Turkey, phone: +90 5071320265, e-mail: aliihsantekin38@hotmail.com 
manipulation of the aorta [4-6]. In contrast to OPCAB, MIDCAB is performed without sternotomy through a small left anterior thoracotomy.

Disease of the left anterior descending artery $(L A D)$, the largest coronary artery, which supplies blood to nearly half of the ventricular myocardium, poses a higher risk for patients compared to diseases of other coronary vessels [7]. Treatment options in isolated LAD stenosis include coronary stenting, percutaneous transluminal coronary angioplasty, and left internal mammary artery (LIMA) bypass grafting. Although the use of coronary stenting for isolated LAD disease has increased in recent years, LIMA to LAD bypass is preferable because of the high long-term patency rates, reduced number of coronary interventions, and high survival rates [8-10]. Surgical treatment can be applied in isolated $L A D$ disease with either OPCAB or MIDCAB. The potential advantages of MIDCAB include reduced surgical trauma, rapid healing, and improved quality of life after surgery [11]. However, MIDCAB is technically challenging since it allows only limited access to the surgical field and limits the exposure of the heart [3].

\section{Aim}

In this study, we compared in-hospital outcomes of patients undergoing surgery for one-vessel coronary artery disease (CAD) with either MIDCAB or OPCAB.

\section{Material and methods}

Patients operated on for one-vessel CAD from October 2013 to December 2015 were retrospectively evaluated in this study. A total of 23 patients who underwent MIDCAB surgery via a left anterior

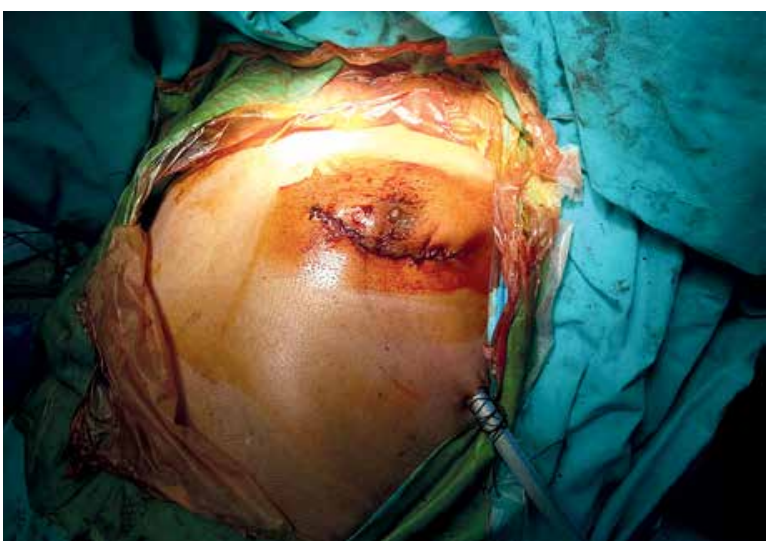

Photo 1. Postoperative appearance of a patient following MIDCAB surgery thoracotomy constituted the MIDCAB group, and 24 patients who underwent $O P C A B$ via a median sternotomy constituted the OPCAB group. Preoperative, intraoperative, and postoperative data of the patients were recorded. Informed consent was obtained from all patients prior to the study.

\section{Patient selection criteria}

The criteria for single vessel revascularization were as follows: a) occlusion of the LAD, b) complex lesions unsuitable for percutaneous coronary angioplasty or stenting, and c) restenosis after angioplasty and stenting. Patients who required additional surgical treatments for diseases other than coronary lesions (i.e., valvular diseases) were excluded. Patients were assigned to study groups according to their general condition, anatomic characteristics, location and type of lesion, and comorbidities. Patients who were scheduled for MIDCAB underwent electron beam computed tomography (CT) in which the distance between the LIMA and the LAD was measured. In patients with an LIMA to LAD distance longer than $7 \mathrm{~cm}$, an $O P C A B$ with sternotomy was performed. Patients were informed about the indications and limitations of MIDCAB, and the final decision regarding the surgery was left up to them.

\section{Surgical technique}

\section{Minimally invasive direct coronary bypass}

Patients were placed in a supine position and intubated with a double-way endotracheal tube. After an $8 \mathrm{~cm}$-long submammary skin incision, the pleural space was entered through the left $4^{\text {th }}$ intercostal space, and the left lung was deflated. With the help of an LIMA retractor, a pedicled LIMA graft was harvested from the $1^{\text {st }}$ intercostal space (proximal end) to the bifurcation of the musculophrenic artery and superior epigastric artery (distal end). Following heparinization, the distal anastomosis was established with the help of a stabilizer and intracoronary shunts while the heart was still beating. A thorax drain was placed into the left thorax. After performing bleeding control, the thoracotomy was closed in the standard fashion (Photo 1).

\section{Off-pump coronary artery bypass}

Patients were positioned on the operation table in the supine position. Standard median sternotomy 
was carried out in all patients. The LIMA was prepared as a pedicled graft using electrocautery and an asymmetric sternum retractor. After heparinization, the pericardium was opened, and the heart was positioned by placing hot wet gauzes under it. A traction suture was placed with 4/0 polypropylene to the proximal part of the LAD. Stabilization was maintained with a vacuum stabilizer. A bloodless operation field was achieved with air/saline insufflation. In cases with high retrograde bleeding, another traction suture was placed with 4/0 polypropylene to the distal part of the LAD. After anastomosis, bleeding control was performed. The sternotomy was closed in the standard fashion after placing a thorax drain (36 Fr) in the left thorax.

\section{Statistical analysis}

Data were presented as $n(\%)$ or mean \pm standard deviation (SD). We used the $\chi^{2}$ test for the analysis of categorical variables and Student's $t$-test for the analysis of continuous variables. We analyzed the data with the SPSS program (SPSS Inc., Chicago, IL, USA). We accepted a $p$-value below 0.05 as statistically significant.

\section{Results}

Overall, 47 patients (11 females, 36 males) were operated on for one-vessel CAD throughout the study period. There were 23 patients ( 7 females, 16 males) in the MIDCAB group and 24 patients (4 females,
20 males) in the OPCAB group. The preoperative characteristics of patients are presented in Table I. Study groups were similar regarding age, gender, history of myocardial infarction, ejection fraction, and comorbidities (Table I).

The same surgical team operated on all patients. Mortality was not observed in either group in the intraoperative or postoperative periods. All patients were operated on under elective conditions. None of the patients required a cardiopulmonary bypass. There was no conversion to sternotomy in the MIDCAB group.

The postoperative data are presented in Table II. Duration of mechanical ventilation $(5.1 \pm 0.7 \mathrm{~h}$ vs. $6.6 \pm 0.9 \mathrm{~h})$, intensive care unit stay $(19.4 \pm 2.5 \mathrm{~h}$ vs. $45.8 \pm 5.4 \mathrm{~h})$, and hospital stay $(4.3 \pm 0.4$ days vs. 5.6 \pm 0.8 days) were significantly shorter in the MIDCAB group compared to those in the OPCAB group ( $p<$ $0.01)$. Patients in the OPCAB group required significantly more blood transfusions (1.83 \pm 0.38 units vs. $0.17 \pm 0.38$ units) and fresh frozen plasma use (2.33 \pm 0.96 units vs. $0.69 \pm 0.76$ units) compared to patients in the MIDCAB group $(p<0.01)$. Hemoglobin on postoperative day one was significantly higher in the MIDCAB group $(11.5 \pm 0.8 \mathrm{~g} / \mathrm{dl})$ compared to that in the OPCAB group $(9.9 \pm 0.4 \mathrm{~g} / \mathrm{dl})(p<0.01)$. The amount of postoperative drainage was $534.7 \pm 97$ $\mathrm{ml}$ in the MIDCAB group and $708.3 \pm 77.5 \mathrm{ml}$ in the OPCAB group $(p<0.01)$.

Atrial fibrillation developed in 2 patients in the MIDCAB group and 1 patient in the OPCAB group

Table I. Preoperative characteristics of patients

\begin{tabular}{|c|c|c|c|}
\hline Parameter & $\begin{array}{l}\text { MIDCAB } \\
(n=23)\end{array}$ & $\begin{array}{l}\text { OPCAB } \\
(n=24)\end{array}$ & $P$-value \\
\hline Age [years] & $56.5 \pm 11.5$ & $53.8 \pm 12.8$ & $>0.05$ \\
\hline Gender (f/m) & $7 / 16$ & $4 / 20$ & $>0.05$ \\
\hline Previous myocardial infarction & $9(39)$ & $6(25)$ & $>0.05$ \\
\hline Ejection fraction (\%) & $41.6 \pm 8.4$ & $41.2 \pm 6.4$ & $>0.05$ \\
\hline Diabetes & $4(17)$ & $8(33)$ & $>0.05$ \\
\hline Hypertension & $20(86)$ & $20(83)$ & $>0.05$ \\
\hline Chronic obstructive pulmonary disease & $2(9)$ & $2(8)$ & $>0.05$ \\
\hline Chronic renal failure & $3(14)$ & $1(4)$ & $>0.05$ \\
\hline Dyslipidemia & $8(35)$ & $10(42)$ & $>0.05$ \\
\hline Hemoglobin [g/dl] & $13.2 \pm 1.1$ & $13.6 \pm 1.3$ & $>0.05$ \\
\hline
\end{tabular}

Data are presented as $n(\%)$ or mean $\pm S D$. 
Table II. Postoperative data of patients

\begin{tabular}{|lccc|}
\hline Parameter & MIDCAB & OPCAB & $P$-value \\
\hline Mechanical ventilation duration [h] & $5.1 \pm 0.7$ & $6.6 \pm 0.9$ & $<0.01$ \\
\hline Length of ICU stay [h] & $19.4 \pm 2.5$ & $45.8 \pm 5.4$ & $<0.01$ \\
\hline Length of hospitalization [days] & $4.3 \pm 0.4$ & $5.6 \pm 0.8$ & $<0.01$ \\
\hline Blood transfusion [units] & $0.17 \pm 0.38$ & $1.83 \pm 0.38$ & $<0.01$ \\
\hline Fresh frozen plasma use [units] & $0.69 \pm 0.76$ & $2.33 \pm 0.96$ & $<0.01$ \\
\hline Hemoglobin on postop day 1 [mg/dl] & $11.5 \pm 0.8$ & $9.9 \pm 0.4$ & $<0.01$ \\
\hline Postoperative atrial fibrillation & $2(9)$ & $1(4)$ & 0.609 \\
\hline Drainage [ml] & $534.7 \pm 97$ & $708.3 \pm 77.5$ & $<0.01$ \\
\hline
\end{tabular}

Data are presented as $n(\%)$ or mean $\pm S D$.

$(p=0.609)$. Sinus rhythm was restored with amiodarone infusion in these patients. None of the patients required reoperation due to bleeding. There was no mortality, conversion to cardiopulmonary bypass, or serious complications (gastrointestinal bleeding, cerebrovascular event, new renal failure, or surgical site infection) in either group. Wound revision was not required in any of the patients.

\section{Discussion}

In this study, we found that, compared to the patients operated on with OPCAB surgery, patients operated with MIDCAB surgery had significantly shorter ventilation time, had fewer intensive care unit and hospital stays, and needed fewer blood transfusions.

Although the number of patients undergoing coronary artery bypass grafting decreases every year, the percentage of patients operated on with offpump techniques, which result in significantly lower in-hospital mortality rates compared to conventional coronary artery bypass techniques, has increased gradually [1]. Similar to other surgical fields, there is an increasing trend in cardiovascular surgery to minimize the trauma caused by surgical intervention itself in order to reduce perioperative mortality after cardiovascular interventions [12]. Minimally invasive techniques such as MIDCAB were developed for this purpose $[5,12]$. MIDCAB, which is carried out with a small, anterior thoracotomy, is the treatment of choice in patients with isolated LAD lesions in some centers. It has also been used for the treatment of coronary disease involving multiple vessels $[4,13,14]$.

The primary goal of MIDCAB is to achieve a graft patency rate similar to that of conventional bypass and to avoid repeated revascularizations. Its secondary goals are to reduce postoperative pain and discomfort and provide a rapid return to active life. Last but not least, MIDCAB has economic goals: shortening the length of hospitalization and reducing costs [12]. Accomplishing these goals is dependent on performing an effective coronary anastomosis and avoiding cardiopulmonary bypass and sternotomy.

Currently, off-pump coronary revascularization on the beating heart is performed worldwide. Patel et al. [15] defined the characteristics of an ideal patient for beating-heart surgery as follows: a) distal vessel diameter of $>1.5 \mathrm{~mm}, \mathrm{~b}$ ) favorable ventricular function, c) hemodynamic stability, d) anterior wall anastomosis, i.e., the LAD artery or the diagonal artery, and e) $<6.5 \mathrm{~cm}$ distance between the posterior table of the sternum fully exposed by the mammary retractor and the pericardium over the $\angle A D$ area. While the standard incision in classical OPCAB surgery is the median sternotomy, technological developments have enabled different approaches to be used for beating-heart surgery [15]. It is important to note that OPCAB and MIDCAB are technically challenging and that both techniques require teamwork for better outcomes. Cuenca et al. recommended intraoperative Doppler evaluation of anastomosis patency due to the technical difficulty of the OPCAB technique [5]. During the surgery, it is important to keep the heart warm and to avoid unnecessary contact with the heart. Cardiac stabilizing devices and pericardial traction sutures are used to place the heart in the correct position to access the vessels on the lateral and posterior surfaces [5]. The stabilizers should not be moved once positioned. 
The LIMA-to-LAD anastomosis is performed first, and then the anastomoses of other vessels are performed, if necessary. Furthermore, anesthetic management of the patient is important to ensure a desirable outcome of OPCAB surgery. The surgeon and the anesthesiologist should work as a team to ensure a safe operation.

Some studies have compared the outcome of patients revascularized with either MIDCAB or OPCAB. Gersbach et al. reported their initial experience with MIDCAB and compared both perioperative and midterm results of MIDCAB and OPCAB [16]. They found that although MIDCAB patients had a shorter intubation time, they received significantly less anastomosis and had a higher rate of serious complications (7 vs. 0) and more discomfort at three months. They concluded that, despite the cosmetic advantages of MIDCAB, OPCAB is more convenient both for the patient and the surgeon [16]. In a larger case series, Detter et al. compared MIDCAB $(n=111)$ performed for LAD stenosis and OPCAB $(n=229)$ carried out for a variety of vessel diseases [17]. The postoperative total graft patency rate was similar between the groups. However, there were three in-hospital deaths in the OPCAB group and none in the MIDCAB group. They concluded that, even though MIDCAB is a challenging technique, it can be performed safely in selected patients with suitable coronary anatomy [17]. With increasing experience, recent studies tend to report a more favorable outcome with MIDCAB compared to OPCAB. In a prospective study, Karpuzoglu et al. compared early results of patients operated on for isolated LAD disease with either MIDCAB or OPCAB [18]. They found that MIDCAB significantly reduced the duration of both mechanical ventilation and hospital stay compared to OPCAB [18]. Similarly, Birla et al. found that patients who underwent LIMA to LAD bypass with MIDCAB had a shorter hospitalization period and a reduced need for blood transfusion; however, other early outcomes, such as mortality, recurrent myocardial infarction, and postoperative stroke, were similar in patients operated on with MIDCAB and OPCAB [19]. In addition, similarly to our results, Zhang et al. reported reduced ventilation duration, intensive care unit stay, hospital stay, and blood transfusion with MIDCAB in comparison to OPCAB [20].

However, Rogers et al. demonstrated in a randomized controlled trial that coronary artery bypass grafting through a small thoracotomy (ThoraCAB) does not improve perioperative or mid-term outcomes compared to OPCAB through a median sternotomy [3]. Furthermore, the average cost of ThoraCAB was $10 \%$ more than OPCAB. However, $65 \%$ of the patients in their study had triple vessel disease, which underlines the importance of correct patient selection for MIDCAB. Multivessel disease is an independent prognostic factor for mortality after MIDCAB [13, 14]. Further studies are needed to define the patient groups that would benefit the most from MIDCAB.

The patency of grafts after MIDCAB has been investigated in some studies. Mack et al. demonstrated that the grafts were patent in $99 \%$ of 103 cases in the angiography performed immediately after the MIDCAB surgery [21]. Some studies show the superiority of MIDCAB over percutaneous coronary intervention in proximal LAD lesions [10]. The MIDCAB is also better than drug-eluting stents concerning the early patency rate and repeated intervention on a target vessel [22]. In a large study including 1,300 patients, Holzhey et al. reported a $94.5 \%$ graft patency rate after 6 months, $91.9 \%$ after 1 year, and 90\% after 7 years [23]. Moreover, they also determined a 5 -year survival rate of $91.9 \%$ and a 7 -year survival rate of $89.4 \%$ following MIDCAB for revascularization of LAD. These patency rates are similar to those achieved with conventional bypass surgery.

Another important advantage of MIDCAB is the reduced frequency of major perioperative complications. Diegeler et al. found that, while postoperative pain was higher in the MIDCAB group compared to the OPCAB group until postoperative day four, the MIDCAB group had less pain, and patients were more comfortable during physical activities after postoperative day 4 [24].

The limitations of our study include the relatively small number of participants, the inclusion of patients with one-vessel CAD, and the lack of long-term outcome data. However, the scarcity of studies in the literature that compare MIDCAB to OPCAB and the need for such studies are factors that contribute significant value to our study.

\section{Conclusions}

We believe that the MIDCAB technique is more advantageous in the early in-hospital period than the OPCAB technique for the treatment of patients with a critical LAD lesion. Further studies compar- 
ing the long-term outcomes of patients treated with MIDCAB or OPCAB are needed.

\section{Conflict of interest}

The authors declare no conflict of interest.

\section{References}

1. Beckmann A, Funkat AK, Lewandowski J, et al. German Heart Surgery Report 2015: The Annual Updated Registry of the German Society for Thoracic and Cardiovascular Surgery. Thorac Cardiovasc Surg 2016; 64: 462-74.

2. Amano A, Hirose H, Takahashi A, Nagano N. Off-pump coronary artery bypass. Mid-term results. Jpn I Thorac Cardiovasc Surg 2001; 49: 67-78.

3. Rogers CA, Pike K, Angelini GD, et al. An open randomized controlled trial of median sternotomy versus anterolateral left thoracotomy on morbidity and health care resource use in patients having off-pump coronary artery bypass surgery: the Sternotomy Versus Thoracotomy (STET) trial. J Thorac Cardiovasc Surg 2013; 146: 306-16.e1-9.

4. Calafiore AM, Giammarco GD, Teodori G, et al. Left anterior descending coronary artery grafting via left anterior small thoracotomy without cardiopulmonary bypass. Ann Thorac Surg 1996; 61: 1658-63; discussion 64-5.

5. Cuenca J, Bonome C. Off-pump coronary artery bypass grafting and other minimally invasive techniques. Rev Esp Cardiol 2005; 58: 1335-48.

6. Diegeler A, Thiele H, Falk V, et al. Comparison of stenting with minimally invasive bypass surgery for stenosis of the left anterior descending coronary artery. N Engl J Med 2002; 347: 561-6.

7. Klein LW, Weintraub WS, Agarwal JB, et al. Prognostic significance of severe narrowing of the proximal portion of the left anterior descending coronary artery. Am J Cardiol 1986; 58: 42-6.

8. Loop FD, Lytle BW, Cosgrove DM, et al. Influence of the internal-mammary-artery graft on 10-year survival and other cardiac events. N Engl J Med 1986; 314: 1-6.

9. Cameron A, Davis KB, Green G, Schaff HV. Coronary bypass surgery with internal-thoracic-artery grafts-effects on survival over a 15-year period. N Engl I Med 1996; 334: 216-9.

10. O'Keefe JH Jr, Kreamer TR, Jones PG, et al. Isolated left anterior descending coronary artery disease: percutaneous transluminal coronary angioplasty versus stenting versus left internal mammary artery bypass grafting. Circulation 1999; 100 (19 Suppl): II114-8.

11. Al-Ruzzeh S, Mazrani W, Wray J, et al. The clinical outcome and quality of life following minimally invasive direct coronary artery bypass surgery. J Card Surg 2004; 19: 12-6.

12. Duhaylongsod FG. Minimally invasive cardiac surgery defined. Arch Surg 2000; 135: 296-301.

13. Sorm Z, Harrer J, Voborník M, et al. Early and long-term results of minimally invasive coronary artery bypass grafting in elderly patients. Kardiol Pol 2011; 69: 213-8.

14. Kofidis T, GerdPaeschke H, Lichtenberg A, et al. Factors affecting post minimally invasive direct coronary artery bypass grafting incidence of myocardial infarction, percutaneous transluminal coronary angioplasty, coronary artery bypass grafting and mortality of cardiac origin. Interact Cardiovasc Thorac Surg 2009; 8: 49-53.

15. Patel AN, Benetti F, Hamman B. Patient selection and technical considerations for off-pump coronary surgery. Proc (Bayl Univ Med Cent) 2003; 16: 291-3.

16. Gersbach P, Imsand C, von Segesser LK, et al. Beating heart coronary artery surgery: is sternotomy a suitable alternative to minimal invasive technique? Eur J Cardiothorac Surg 2001; 20: 760-4.

17. Detter C, Reichenspurner H, Boehm DH, et al. Minimally invasive direct coronary artery bypass grafting (MIDCAB) and off-pump coronary artery bypass grafting (OPCAB): two techniques for beating heart surgery. Heart Surg Forum 2002; 5 : 157-62.

18. Karpuzoglu OE, Ozay B, Sener T, et al. Comparison of minimally invasive direct coronary artery bypass and off-pump coronary artery bypass in single-vessel disease. Heart Surg Forum 2009; 12: E39-43.

19. Birla R, Patel P, Aresu G, Asimakopoulos G. Minimally invasive direct coronary artery bypass versus off-pump coronary surgery through sternotomy. Ann R Coll Surg Engl 2013; 95: 481-5.

20. Zhang L, Cui Z, Song Z, et al. Minimally invasive direct coronary artery bypass for left anterior descending artery revascularization - analysis of 300 cases. Videosurgery Miniinv 2016; 10 : 548-54.

21. Mack MJ, Magovern JA, Acuff TA, et al. Results of graft patency by immediate angiography in minimally invasive coronary artery bypass surgery. Ann Thorac Surg 1999; 68: 383-9; discussion 389-90.

22. Benedetto U, Raja SG, Soliman RF, et al. Minimally invasive direct coronary artery bypass improves late survival compared with drug-eluting stents in isolated proximal left anterior descending artery disease: a 10-year follow-up, single-center, propensity score analysis. J Thorac Cardiovasc Surg 2014; 148: 1316-22.

23. Holzhey DM, Jacobs S, Mochalski M, et al. Seven-year-follow-up after minimally invasive direct coronary artery bypass: experience with more than 1300 patients. Ann Thorac Surg 2007; 83: 108-4.

24. Diegeler A, Walther T, Metz S, et al. Comparison of MIDCAP versus conventional CABG surgery regarding pain and quality of life. Heart Surg Forum 1999; 2: 290-5.

Received: 17.02.2017, accepted: 14.04.2017 